\title{
ESTILOS DE ENSEÑANZA DE DOCENTES VINCULADOS A LA LICENCIATURA EN DEPORTE Y SU RELACIÓN CON EL LOGRO ACADÉMICO DE LOS ESTUDIANTES
}

\author{
TEACHING STYLES OF PROFESSORS FROM THE BACHELOR IN SPORTS AND
} ITS RELATIONSHIP WITH THE ACADEMIC ACHIEVEMENT OF STUDENTS

\author{
ESTILOS DE ENSINO DOS PROFESSORES DA LICENCIATURA EM ESPORTES E \\ SUA RELAÇÃO COM AS CONQUISTAS ACADÊMICAS DOS ESTUDANTES
}

\author{
Víctor H. Durán Camelo ${ }^{1}$ \\ Efraín Serna Caldas ${ }^{2}$ \\ Luis Alberto Rodríguez ${ }^{3}$
}

Resumen

El artículo tiene como propósito mostrar los avances investigativos del proyecto: Estilos de enseñanza de
docentes vinculados al programa de deporte en la Universidad Pedagógica Nacional y su relación con el logro
académico de los estudiantes. La metodología se sustenta en la investigación cualitativa de tipo interpretati-
va-descriptiva. En su primera fase, contrasta los resultados del Cuestionario sobre la orientación del profesor
universitario (coDPu) de Feixas (2006) aplicado de manera censal, con el resultado de entrevistas semies-
tructuradas aplicadas a un grupo focal de maestros de la licenciatura. Se asume como sujetos de investigación
a 36 docentes que orientan los espacios académicos en la Licenciatura en Deporte para llegar a una muestra
(grupo focal) que permita profundizar en la comprensión cualitativa de sus estilos de enseñanza. Se espera que
los resultados permitan aclarar el panorama de indagación señalado en por Durán, Pedraza y Serna, , (2014),
sobre los factores que intervienen en el logro académico de los estudiantes de licenciatura y el grado de inje-
rencia, que en este proceso, tiene el docente, las didácticas específicas y su estilo de enseñanza, en este proceso.

Palabras clave: estilos de enseñanza; logro académico; didácticas específicas; formación de licenciados

1 Doctorando en Educación, magíster en Educación, especialista en Pedagogía, licenciado en Educación Física, docente-investigador (ocasional y tiempo completo) de la Universidad Pedagógica Nacional-Facultad de Educación Física. Correo electrónico: vhduran@pedagogica.edu.co

2 Magíster en Educación de Adultos, licenciado en Ciencias de la Educación, docente de planta de la Universidad Pedagógica Nacional. Correo electrónico: efserna@pedagogica.edu.co

3 Especialista en Pedagogía del Entrenamiento Deportivo, licenciado en Educación Física, docente (ocasional y tiempo completo) de la Universidad Pedagógica Nacional, coordinador del programa Licenciatura en Deporte. Correo electrónico: Irodriguez@pedagogica.edu.co 


\begin{abstract}
The purpose of the paper is to show the research advances of the project "Teaching Styles of Professors from the Bachelor in Sports of Universidad Pedagógica Nacional and its Relationship with the Academic Achievement of Students." The methodology is based on a qualitative research of interpretative-descriptive nature. In the first part, we compare the results of Feixas' (2006) Questionnaire for the Analysis of the University Teachers' Teaching Orientation (CODPU) applied using the census method, with the result of semi-structured interviews applied to a focus group of professors from the Bachelor program. The research subjects consists of 36 professors from the Bachelor in Sports, thus making a sample (focus group) that that allows us to delve into the qualitative understanding of their teaching styles. These results are expected to clarify the panorama of inquiry indicated by Durán, Pedraza, and Serna (2014) about the factors intervening in the academic achievement of undergraduate students and the teacher's degree of interference in this process, as well as the specific didactics and their teaching style in this process.
\end{abstract}

Keywords: teaching styles; academic achievement; specific didactics; training of graduates

\title{
Resumo
}

0 artigo tem como objetivo apresentar os avanços do projeto de pesquisa: Estilos de ensino dos professores da Licenciatura em Esportes da Universidade Pedagógica Nacional e sua relação com as conquistas acadêmicas dos estudantes. A metodologia utilizada foi a pesquisa qualitativa do tipo descritivo-interpretativo. Em sua primeira fase, contrastamos os resultados do Questionário sobre a orientação do professor universitário (CODPU) de Feixas (2006) aplicado como censo, com os resultados das entrevistas semiestruturadas aplicadas a um grupo focal de professores do programa. Os sujeitos da pesquisa foram 36 professores que ministram aulas na Licenciatura em Esporte, dos que selecionamos uma amostra (grupo focal) que possibilitou a compreensão qualitativa de seus estilos de ensino. Esperamos que os resultados permitam uma visão mais clara da problemática assinalada por Durán, Pedraza e Serna, (2014), sobre os fatores envolvidos na conquista acadêmica dos estudantes de graduação e o grau de interferência do professor, seu estilo de ensino e as didáticas específicas neste processo.

Palavras-chave: estilos de ensino; conquista acadêmica; didática específica; formação de professores

Fecha de recepción: 18 de octubre de 2016

Fecha de aprobación: 30 de agosto de 2017

Para citar este artículo:

Durán, V., Serna,E., Rodríguez, L. (2017). Estilos de enseñanza de docentes vinculados a la licenciatura en deporte y su relación con el logro académico de los estudiantes. Lúdica Pedagógica, 26, 71-82. 


\section{INTRODUCCIÓN}

El proyecto se enmarca en el desarrollo teórico del grupo de investigación denominado Gestión y Pedagogía de la Actividad Física y el Deporte, consolidado a partir de la indagación sobre procesos de actividad física y el deporte social comunitario, actualmente, en la comprensión de los estilos de enseñanza-aprendizaje que se configuran en el planteamiento curricular para sujetos, contexto y disciplina particular, como son los estudiantes y docentes del programa de Licenciatura en Deporte, en la Facultad de Educación Física de la Universidad Pedagógica Nacional.

Los avances investigativos han sido socializados en distintos eventos de orden nacional e internacional, entre ellos, el Congreso Internacional Expo Motricidad, el Congreso Internacional de Educación (CIDE-Ecuador), y el Congreso Internacional Alesde, en su versión 2014 y 2016, así como la publicación de artículos académicos, entre ellos, Durán (2010, 2011a, 2011b, 2012); Calderón y Álzate (2010); Calderón, Puentes, Durán, Alzate y Barinas (2012); Durán y Castro (2013). Durán, Pedraza y Serna (2014), entre otro tipo de producción académica como talleres comunitarios, diplomados y proyectos de intervención y extensión llevados a cabo por los autores desde la Facultad de Educación Física.

En términos prospectivos, se comprende que el impacto social del licenciado en Deporte se encuentra condicionado por las transformaciones curriculares generadas desde el programa de Deporte, en torno a los contenidos, construcción de saberes, la manifestación de competencias docentes, investigativas y políticas, así como la construcción de un sentido social expreso en el rol y pensamiento de maestro.

Puesto que la intención es rastrear los factores que condicionan el logro académico del estudiante, se ha consolidado de manera estratégica una mirada amplia y a largo plazo que da la posibilidad de postular nuevas categorías y rutas de indagación en torno a la problemática. La teoría consultada muestra una fuerte correlación entre el estilo de enseñanza particular y el logro académico del estudiante (Laudadío, 2012a), sirviendo como sustento a la pregunta por el rendimiento académico desde el enfoque de la enseñanza, explícito en los objetivos del proyecto para la vigencia 2016-1 y 2016-2.
Por lo tanto, el proyecto de investigación "Estilos de enseñanza de docentes vinculados al programa de deporte y su relación con el logro académico de los estudiantes" da continuidad a la necesidad institucional de explorar los procesos metateóricos internos referentes a la enseña-aprendizaje, teniendo en cuenta las características, calidad y efectividad de las didácticas específicas desarrolladas por los docentes.

\section{Planteamiento del problema}

La consolidación de programas de licenciatura en la Universidad Pedagógica Nacional (UPN) en vía de acreditación de calidad requiere de procesos internos de autoevaluación e investigación que contribuyan al mejoramiento de aspectos académico-administrativos, para evidenciar cómo los procesos de enseñanza aprendizaje, las propuestas metodológicas y demás condiciones asociadas, hacen posible el cumplimiento de los objetivos del programa y la consecución del perfil del egresado propuesto por los programas. No obstante, dicho perfil, idealizado por el programa y por la sociedad, depende no solo de la titulación, sino también de la identificación, ajuste o solución de problemáticas presentes en la formación, identificando y afectando a su vez, las posibles causas.

Según se reporta en las evaluaciones internas, la queja generalizada en los actuales espacios académicos universitarios - desde la perspectiva del estudiante- está relacionada con una pérdida de sentido frente al proceso de formación, lo cual redunda en tareas descontextualizadas, abstractas y en últimas no significativas para la vida, insatisfacción manifiesta sobre el proceso de enseñanza aprendizaje, sobre los maestros, los escenarios, medios, contenidos y tareas.

Desde la perspectiva del docente, la problemática se centra en el bajo rendimiento de los estudiantes, en las falencias del proceso de aprendizaje, la poca comprensión manifiesta frente a temáticas complejas, el precario nivel de lectoescritura, el desarrollo de tareas y actividades de manera superficial, bajo resultado en exámenes de contenido de aprendizaje, escasa regulación o autorregulación en las actividades de aprendizaje, entre otras.

Este panorama evidencia el profundo desconocimiento sobre los procesos relacionados con el 
estudiante o el docente en el aula de clase, sobre la forma en que aprenden los estudiantes, sus estilos de aprendizaje y sus intereses; no reconoce, por ejemplo, en qué medida estos dan cuenta de procesos autorreguladores, o en qué medida orientan sus aprendizajes (por metas o autoeficacia), tampoco presentan datos sistematizados sobre el nivel de logro académico alcanzado de manera individual o colectiva, que den cuenta de avances o retrocesos en los objetivos propuestos.

De igual manera sucede en la perspectiva del docente, puesto que los programas académicos no identifican sus estilos de enseñanza, la variedad de didácticas manifiestas que son regidas por la especificidad disciplinar, las ideologías que sustentan las valoraciones del trabajo del estudiante, entre otros aspectos. Sobre todo, muestra, en el caso particular de la Licenciatura en Deporte, una dicotomía entre los aspectos teórico-conceptuales desarrollados en el aula y la aplicación práctica requerida en el campo como competencia del estudiante, lo que deriva en la excesiva didactización en el aula, y compromete procesos de aprendizaje de contenidos. Otra falencia manifiesta es la ausencia de ejercicios exploratorios que permitan comprender la relación entre las metodologías presentes en la Licenciatura en Deporte, propia de los estilos de enseñanza del maestro con sus saberes particulares y los logros académicos relacionados con el estilo de aprendizaje de los estudiantes.

Lo anteriormente planteado deriva en la siguiente pregunta: ¿cuál es el estilo de enseñanza de los docentes del programa de Deporte en la Universidad Pedagógica Nacional? Y ¿qué relación existe entre dichos estilos y el logro académico de los estudiantes de la licenciatura?

\section{MARCO DE REFERENCIA}

\section{Los estilos de enseñanza}

Siguiendo el trabajo de Laudadío (2012a) y Laudadío y Da Dalt (2014), el estudio de los estilos de enseñanza se concentra en la identificación y análisis de las creencias, valores, actitudes, modos de trabajo y habilidades que manifiestan los docentes en el desarrollo de su profesión.
Entonces, el estilo de enseñanza se comprende como la expresión de una construcción didáctica del docente basado en su formación, historia de vida, rutinas establecidas e ideologías, dan cuenta de un modo particular en que este se comporta frente a la intención de enseñar y que se constituye en hábito de su profesión, podría decirse que es una característica medianamente estable y permanente en él.

Por estar sujetos a la construcción particular del sujeto docente, el estilo de enseñanza presenta características singulares, comportamientos, verbalizaciones, acciones y decisiones que le son propias; no obstante, pueden ser catalogadas de manera general como tendencias o estilos en relación con las maneras de enseñar y de establecer relaciones intersubjetivas en el aula.

Según Gayle (citado en Laudadío, 2012a, p. 81): "Entre los aspectos que describen cada estilo no se pueden dejar de considerar: las características personales del profesor, sus modos propios de hacer y los procesos que permiten alcanzar determinados resultados". Este último aspecto es, al parecer, el criterio que más atribuye significatividad a esta toma de decisiones, puesto que, a la luz de lo institucional, a mayor resultado en términos de logro académico del estudiante, mayor será la importancia del estilo de enseñanza y la eficacia didáctica del maestro.

Como ya se ha mencionado, "los estilos de enseñanza manifiestan las preferencias en los modos de enseñar de los educadores, las cuales suponen determinadas creencias y/o concepciones de la enseñanza" (Heimlich y Norland, 2002, citado en Laudadío, 2012a, p. 81), lo que deriva en fundamentos epistemológicos que sustentan, además de la postura frente al conocimiento, unas maneras de hacer para propiciar el aprendizaje del otro que, en términos colectivos, dan cabida al enfoque o estilo de enseñar.

La categorización de diversos estilos ofrece a los educadores el punto de partida para explorar y reflexionar acerca de su propia enseñanza y en última instancia, se refieren a la coherencia entre la concepción teórica y el modo concreto de enseñar. Según estos autores, la búsqueda de la coherencia en la enseñanza exige la reflexión personal de "quién soy y qué creo", a lo que se añade un análisis metacognitivo de cuáles son los objetivos que, 
en la enseñanza concreta, motivan mi estilo propio. (Laudadío, 2012a, p. 81).

En este sentido, el análisis e interpretación de la creencia y el valor implícito en la enseñanza son parte fundamental del proceso, al igual que la identificación de las relaciones entre los anteriormente mencionados y el proceso de enseñanza-aprendizaje.

Según plantea Laudadío (2012a), la identificación del estilo es un medio que permite descubrir aspectos que redunden en la mejora de los modos particulares en que se realiza el trabajo docente cotidiano. También permite reconocer la individualidad e innovación del docente en sus formas de enseñar y de establecer relaciones en el aula, con el fin de ser cada día más eficaz.

En términos de Heimlich y Norland:

Al considerar el concepto de estilos de enseñanza desde el punto de vista teórico, sostienen que estos no se reducen a un solo aspecto, sino que abarcan todo el proceso de enseñanza-aprendizaje. [...] Es decir que, según estos autores, cinco serían los elementos que componen un modelo de enseñanza: el educador, el educando, el grupo, el contenido y el ambiente; y si bien todos están presentes en cualquier acción educativa, puede variar la relación entre ellos y el grado de importancia que se le concede. (Citado en Laudadío, 2012a, p. 81).

Al respecto, Gayle (1994) analiza algunas investigaciones realizadas en las últimas décadas, e identifica dos posturas: por un lado, los que consideran los estilos de enseñanza como mera decisión dicotómica entre estrategias y dimensiones que son tomadas aisladamente (Axelrod, 1973; Bruner, Goodnow y Austin, 1956; Kagan, Moss y Sigel, 1960; citados en Gayle, 1994). Por otro lado, los que discuten los estilos de enseñanza en términos de un perfil relativamente más complejo que el simple uso de estrategias (Gregorc, 1982; Kolb, 1984; Powell, 1984; citados en Gayle, 1994).

Las investigaciones dan cuenta de un enfoque teórico y otro empírico de los estilos de enseñanza, estas se preguntan sobre cómo enseñan los profesores y cuál es la mejor forma de hacerlo:

Estos estudios definen a los estilos de enseñanza como: la elección de cada educador en relación con: sus conductas y creencias educativas (Heimlich y Norland, 2002), los estilos cognitivos, de aprendi- zaje y de pensamiento del educador que influyen en la acción educativa (Zhang, 2001) y las características de la relación que establece el educador con el educando; esto es, los rasgos del vínculo pedagógico. (Cohen y Amidon, 2004, citado en Laudadio y Mazzitelli, 2015, p. 12).

No obstante, la manera más general de abordar la reflexión y la indagación por los estilos de enseñanza se encuentra vinculada a la clasificación: estilos centrados en el profesor y estilos de enseñanza centrados en el estudiante (Carpenter y Tait, 2001; Prosser y Trigwell, 1996).

El estilo centrado en el profesor considera al aprendizaje en términos de cambios de conducta, en el cual el estudiante tiene un papel pasivo (receptor de la información) y el educador asume un rol de transmisión de contenidos al estudiante.

En el estilo centrado en el estudiante la acción docente se encuentra focalizada más en el estudiante y sus necesidades que en el conocimiento que se debe transmitir. Por ello, se aproxima de mejor manera a las metodologías colaborativas y dialógicas (maestro-estudiante), ambos comprendidos como participantes activos del proceso de aprendizaje en general.

Entre las tipologías más complejas en el campo disciplinar de la educación física y el deporte, se encuentra la propuesta de Mosston y Ashworth (1993), quienes describen nueve estilos de enseñanza tomando como criterio de clasificación la toma de decisiones.

Los estilos en mención se organizan desde aquellos en los que el docente es quien toma las decisiones, denominados de enseñanza directiva, a los estilos donde el alumno es el que toma todas las decisiones, o gran parte de ellas, y son llamados de enseñanza no directiva, donde el alumno no solo repite conocimiento, sino que él mismo es responsable de generar nuevos aprendizajes.

Grasha (citado en Vaughn y Baker, 2001) propone una variedad de estilos en función de las necesidades del aprendizaje y sugiere clasificarlos en experto, formal, autoritario, personal, facilitador y delegador; en la descripción de cada estilo considera sus ventajas y desventajas (p. 83).

La importancia de la investigación en estilos de enseñanza radica en la posibilidad de descubrir en el proceso educativo cuáles son las formas de enseñar y 
qué ventajas existen en un determinado modo frente al otro, de igual manera, permite potenciar el proceso educativo tanto para el docente como para el estudiante, lo que dinamiza de manera positiva los procesos y relaciones de aula, y aporta metodológica e investigativamente al campo disciplinar específico desde la puesta en práctica de métodos, técnicas e instrumentos de indagación.

Metodológicamente, se han abordado variedad de cuestionamientos relacionados con el estilo de enseñanza del maestro, que se constituyen en factores o indicadores para su descripción. Según Bain (2007), se definen en la pregunta por qué saben los profesores sobre cómo aprende el estudiante, cómo preparan sus clases, qué esperan los docentes de sus estudiantes, cómo dirigen sus clases, de qué manera es el trato con sus estudiantes y cómo evalúan a sus estudiantes y a sí mismos, vinculando estas preguntas al mejoramiento docente.

Por otra parte, se encuentran los cinco factores propuestos por Gayle (citado en Laudadío y Da Dalt, 2014, p. 487): 1. La personalidad del profesor. 2 . La filosofía educativa que subyace a su práctica docente. 3. Su comportamiento y conocimiento - no solo de la materia, sino también de sus estudiantes y necesidades-. 4. Las estrategias de enseñanza, y 5. Las técnicas utilizadas en la clase.

Por su parte, Grasha (1994) establece factores que determinan el modo de enseñar de los docentes, entre ellos: las capacidades de los estudiantes (conocimientos previos, iniciativas, motivación, responsabilidad, madurez, etc.), las necesidades del docente respecto al control de la clase (organización, presentación de metas por alcanzar, seguimiento del progreso de los estudiantes, determinación o evaluación del nivel de rendimiento, etc.), y la capacidad del profesor para construir y mantener una relación educativa (comunicación bidireccional, escucha atenta, habilidades de comunicación interpersonal).

Según Laudadío (2012a), en relación con los anteriores criterios, la conceptualización de los estilos de enseñanza debe tener en cuenta, de manera general, la visión del profesor, la visión que tiene el docente acerca del estudiante, el fin de la enseñanza perseguida por los profesores, los contenidos de enseñanza y la concepción de la evaluación.
Así las cosas, el proyecto plantea una articulación cualitativa entre indicadores generales y particulares para la identificación de estilos de enseñanza en el maestro de la Licenciatura en Deporte, atendiendo de manera general a lo propuesto por Laudadío (2012b) y Feixas (2006) y, en términos disciplinares, siguiendo las categorías e indicadores para la definición de estilos de enseñanza propuestos por Mosston y Ashworth (1993).

\section{Los estilos de enseñanza y el logro académico}

Uno de los principales cuestionamientos de la academia frente a los procesos de enseñanza está centrado en el rendimiento escolar, pensado más allá de los resultados de la evaluación formal como exclusivo criterio determinante en la constatación de dicho rendimiento, para incluir otros factores como el aprendizaje profundo, nivel de logro alcanzado en materias específicas, tasas de repetición y de retención escolar, entre otros, como afirma Cascón (2000).

No obstante, es la calificación uno de los criterios más objetivos y concretos para indagar los niveles de logro del estudiante, como afirma Cascón (2000, pp. 1-11):

Probablemente unas de las variables más empleadas o consideradas por los docentes e investigadores para aproximarse al rendimiento académico son: las calificaciones escolares; "el indicador del nivel educativo adquirido, en la totalidad de los países desarrollados y en vías de desarrollo, ha sido, sigue y probablemente seguirá siendo las calificaciones escolares. A su vez, éstas son reflejo de las evaluaciones y/o exámenes donde el alumno ha de demostrar sus conocimientos sobre las distintas áreas o materias, que el sistema considera necesarias y suficientes para el desarrollo como miembro activo de la sociedad".

Por otra parte, el problema aborda, de igual manera, las preguntas por los factores que hacen que el logro académico del estudiante se potencie en los escenarios educativos, y en la caracterización de los hábitos y condiciones que harán del estudiante un sujeto exitoso dentro y fuera de la escuela.

Entre las variables del logro académico, se muestran fundamentales los factores socioeconómicos, la amplitud de los programas de estudio, las metodologías de enseñanza utilizadas, la dificultad de emplear 
una enseñanza personalizada, los conceptos previos que tienen los alumnos, así como el nivel de pensamiento formal de los mismos (Benítez, Giménez y Osicka, 2000).

Para Jiménez (2000), el rendimiento escolar es un "nivel de conocimientos demostrado en un área o materia, comparada con la norma de edad y nivel académico", por ello, frente a la pregunta por el logro escolar se establecen, de manera general, dos conjuntos de causas: los aspectos relacionados con la escuela como sistema educativo y aquellas características que los alumnos exhiben a partir de su contexto social, de sus capacidades personales, de sus motivaciones; en otras palabras, se puede establecer, en cuanto al logro académico, factores extrínsecos e intrínsecos tanto a la escuela como al sujeto.

Con base en los resultados de la investigación sobre estilos de aprendizaje (Durán, Serna y Pedraza, 2016), se intenciona las acciones del proyecto hacia el estilo de enseñanza, como factor extrínseco, asociado a al sistema educativo, donde el logro académico no solo es un producto del proceso de formación, sino un ideal del mismo, por lo cual se exaltan las siguientes implicaciones: 1. El logro académico se sustenta en factores múltiples del proceso académico y administrativo. 2. El logro académico pude ser monitoreado en términos generales: el programa, los ciclos de formación o los espacios académicos; o en términos individuales: el maestro y las valoraciones que asigna en su proceso, el estudiante y el producto concreto de su labor en el semestre académico.

\section{Antecedentes investigativos}

Una pregunta obligada de la formación se centra en determinar qué hace que unos (estudiantes) aprendan más y de una mejor manera que otros. Por otra parte, también ha circulado la pregunta por los procesos de orden cognitivo que permitirían aproximar el procesamiento de la información hacia el aprendizaje profundo y significativo, teniendo en cuenta criterios de percepción, emoción y de actuación en el estudiante, sin dejar a un lado la toma de decisiones conceptuales, de estructura teórica, didáctica y metodológica del maestro.

En consecuencia, teorías cognitivas asociadas a la educación e investigaciones sobre estilo cognitivo, estilo de aprendizaje y conocimiento profesional, reconocen en el maestro una serie de saberes, condiciones, fundamentos y experiencias que son puestas en escena en el aula de clase.

De igual manera acontece con los estilos de enseñanza, existe mucha producción en cuanto a instrumentos validados para su indagación, algunos diseñados para identificar las concepciones, preocupaciones, problemas o estrategias didácticas de los profesores universitarios, lo que permite, desde su uso, la comprensión del modo y la orientación de la enseñanza universitaria. Entre estos se encuentran:

- El Effective Teacher Inventory (ETI), elaborado por Zhang (2001) sobre aspectos fundamentales de la enseñanza eficaz, como cualificación académica, preparación en el área de conocimiento, personalidad, relación con los estudiantes, motivación y dinámica de clase.

- Principles of Adult Learning Sacla (PALS), elaborado por Schaefer y Zygmont (2003), utilizado para identificar los estudios de enseñanza, centrado en el estudiante o centrado en el profesor.

- El cuestionario CodPu de Feixas (2002) pretende conocer el estilo de enseñanza del profesor universitario (preocupaciones, concepciones sobre la docencia, dinámica metodológica y evaluación).

Cada uno de ellos con criterios y potencialidades particulares de indagación (Laudadío, 2012b).

Se reconocen y valoran teóricamente otras investigaciones, como la propuesta por Laudadío y Da Dalt (2014) denominada "Estudio de los estilos de enseñanza y estilos de aprendizaje en la universidad", el cual busca conocer cuáles son las formas típicas de enseñanza de los profesores universitarios en relación con las distintas disciplinas de enseñanza, para lo cual utilizaron el coDPU (Feixas, 2006), indagaron a una muestra de 188 docentes de universidades privadas del sistema educativo argentino. Los resultados indican que no se observaron desde el punto de vista descriptivo diferencias en las distintas carreras analizadas, en las distintas áreas de conocimiento se encontraron profesores que adoptan tanto el estilo centrado en el docente como el estilo centrado en el estudiante. 
Por otra parte, Saza y Henao (2012), con el trabajo "Actitudes-estilos de enseñanza: su relación con el rendimiento académico", buscaron determinar las relaciones existentes entre las actitudes y los estilos de enseñanza en 25 docentes de 180 estudiantes con alto rendimiento académico. Los resultados revelaron que las actitudes y los estilos de enseñanza que tienen más presencia son participación democrática, importancia social del trabajo docente, mejoramiento profesional, estilo que propicia la socialización, estilo que posibilita la participación y estilo que favorece la creatividad.

Por último, la investigación de Hernández y Abello (2013), denominada "Los estilos de enseñanza de los docentes de la Licenciatura en Educación con énfasis en Educación Especial de la Universidad Pedagógica Nacional", refleja la caracterización de los docentes a partir del modelo teórico e instrumental de estilo de enseñanza de Abello, Hernández y Hederich (2011). Se aplicó el inventario de estilo de enseñanza versión 3.4 a 19 docentes, caracterizados por 622 estudiantes en 39 asignaturas de la licenciatura. La caracterización se realizó a partir de las dimensiones que estructuran el modelo, identificando los aspectos estables y variables en el estilo de enseñanza de un mismo docente en diferentes asignaturas y explorando las relaciones entre el tipo, nivel de formación y tiempo de experiencia de los docentes y el estilo de enseñanza.

Es evidente que la temática propuesta es objeto de estudio y preocupación de la academia en todos los niveles del sistema educativo, por cuanto la caracterización y comprensión de los estilos de enseñanza y su incidencia en el logro del estudiante puede contener la clave de la transformación educativa y obtención de la calidad, por lo menos en términos del aprendizaje, es decir, procesos de aprendizaje profundo y significativo en el estudiante.

\section{METOdOLOGÍA}

El proceso se asume desde la investigación cualitativa de tipo interpretativo y descriptivo, seleccionando una población conformada por 36 docentes que hacen parte de la Licenciatura en Deporte, a los cuales se les aplicó el cuestionario denominado: "Cuestionario sobre orientación docente del profesor universitario" (CODPU), Feixas (2002).

La muestra principal derivó en un grupo focal de 10 docentes, escogido entre aquellos docentes que, según Feixas (2002), presentaron un estilo orientado hacia la enseñanza, teniendo en cuenta criterios "disciplinares" en relación con las áreas de formación, atendiendo a las didácticas particulares manifiestas.

La pretensión de la primera fase se centró en caracterizar el estilo de enseñanza y clasificar la orientación de enseñanza del maestro. En una fase posterior, encontrar correlación entre el estilo del maestro y el logro académico de los estudiantes.

\section{Instrumento y procedimientos}

Para la investigación se contrastó el resultado del instrumento CoDPu (Feixas, 2002) en su versión resumida, con entrevistas semiestructuradas aplicadas a grupo focal, buscando identificar el estilo de enseñanza del profesor universitario y su correspondencia con el logro académico del estudiante.

El instrumento coDPu está compuesto por 36 ítems que evalúan distintos aspectos de la docencia universitaria, 18 de sus ítems caracterizan en el docente un estilo transmisivo centrado en el profesor como protagonista en el aula. Los 18 ítems restantes representan un estilo participativo y reflexivo, donde el estudiante se torna activo en el proceso, modificando el rol del docente, quien se torna un guía o colaborador que ayuda en el desarrollo de las ideas del estudiante.

Cada orientación es definida a partir de cinco aspectos: (a) las características del profesor, (b) las preocupaciones del profesor, (c) el dominio del contenido, (d) el proceso de enseñanza y aprendizaje (concepciones y estrategias docentes) y (e) la relación con los estudiantes (tabla 1).

Es importante aclarar que la implementación del CODPU se realizó con la pretensión de identificar dos enfoques u orientaciones docentes: uno centrado en el profesor y su enseñanza y otro centrado en el aprendizaje de sus estudiantes, para constituir, posteriormente, el grupo focal que fue entrevistado. 
Tabla 1. Ejemplo instrumento coDPU

\begin{tabular}{|l|l|l|l|l|l|}
\hline $\begin{array}{l}\text { Cuestionario sobre la orientación docente del } \\
\text { profesor universitario }\end{array}$ & $\begin{array}{c}\text { Totalmente } \\
\text { de acuerdo } \\
\text { de los estudiantes }\end{array}$ & $\mathbf{5}$ & $\mathbf{3}$ & $\mathbf{2}$ & $\begin{array}{c}\text { Totalmente } \\
\text { en desacuerdo }\end{array}$ \\
\hline $\begin{array}{l}\text { 1. Soy paciente y sensible a las preocupaciones } \\
\text { de }\end{array}$ & & & & & \\
\hline $\begin{array}{l}\text { 2. Procuro que los estudiantes acaben el curso } \\
\text { con unos buenos apuntes }\end{array}$ & & & & & \\
\hline $\begin{array}{l}\text { 3. Me interesa saber qué y cómo aprenden los } \\
\text { estudiantes }\end{array}$ & & & & & \\
\hline 4. Me gusta ser el protagonista de la clase & & & & \\
\hline $\begin{array}{l}\text { 5. Me preocupa como puedo ayudar a aprender } \\
\text { a los estudiantes }\end{array}$ & & & & & \\
\hline $\begin{array}{l}\text { 6. Me preocupa la opinión y la impresión que } \\
\text { pueda causar en los estudiantes }\end{array}$ & & & & & \\
\hline $\begin{array}{l}\text { 7. Me preocupa como cubrir las necesidades } \\
\text { educativas de cada estudiante }\end{array}$ & & & & & \\
\hline $\begin{array}{l}\text { 8. Me preocupa que los estudiantes no adquieran } \\
\text { los principales conceptos de la asignatura }\end{array}$ & & & & & \\
\hline $\begin{array}{l}\text { 9. Justifico a mis estudiantes la razón de mis } \\
\text { decisiones docentes }\end{array}$ & & & & \\
\hline $\begin{array}{l}\text { 10. Hago todo lo posible para poder abordar todo } \\
\text { el programa }\end{array}$ & & & & & \\
\hline $\begin{array}{l}\text { 11. Animo a los estudiantes a hacer tutorías para } \\
\text { hablar de sus dificultades con la asignatura }\end{array}$ & & & & & \\
\hline
\end{tabular}

Fuente: Feixas, 2002.

\section{RESULTADOS}

Los resultados del proceso de indagación indican lo siguiente:

Existen factores asociados al estilo de enseñanza del docente que determina la manera en que este gestiona su espacio académico y asigna valor cualitativo y cuantitativo a las acciones del estudiante, promoviendo o limitando aquello que consideramos logro académico.

Estos factores determinan el grado de importancia que se le asigna a los espacios académicos y a las disciplinas que las sustentan (encontrándose que para efectos del logro académico, existen unos espacios más importantes que otros), con lo que se hacen relevantes aquellos que valoran o puntúan más bajo el trabajo estudiantil, por ser talanqueras para la promoción y para la obtención de promedios deseables, en este caso particular. Se evidencia que los espacios académicos "no disciplinares" mantienen el promedio de notas más bajas.

En cuanto a los docentes se identifica, desde la aplicación del CODPU, una tendencia de orientación hacia el aprendizaje, es decir, que su énfasis se encuentra aprenda y asuma un rol activo en el proceso de enseñanza-aprendizaje, sin embargo, el grupo de profesores manifestó correspondencias con una amplia gama de posibilidades de enseñanza, en términos de lo que Mostton (1993) denomina el espectro de los estilos de enseñanza no directivos. 
Por consiguiente, el logro académico, desde una mirada general, estaría dependiendo principalmente de la disciplina de formación del docente (disciplinar o no disciplinar), el nivel de formación y la personalidad del docente, no tanto del estilo de enseñanza manifiesto.

En cuanto a la formación disciplinar, existe tendencia a valorar de mejor manera al estudiante (notas altas), cuando el docente presenta formación en disciplinas del área humanística o en las didácticas del deporte. En contraposición, la nota del estudiante presenta tendencias bajas cuando la formación del maestro es no disciplinar, principalmente en asignaturas referidas al lenguaje, estadística y ciencias biológicas.

El nivel de formación se observa como determinante del logro académico, dado que la evidencia muestra que existe menor exigencia por parte de los maestros en el ciclo de fundamentación, muy por el contrario de lo que acontece en el ciclo de profundización en donde las notas tienden a la baja.

De igual forma, la personalidad del docente es determinante pues, en primera instancia, algunos maestros refieren la existencia de condiciones sociales y personales del estudiante, que median los criterios de valoración y asignación de la nota. En segunda instancia, la empatía, la consideración, la afectividad, al igual, que las diferencias, el rechazo o la predisposición hacia el estudiante, alteran de manera positiva o negativa la asignación de la nota, por ende, la acumulación de promedios en el proceso de formación.

Si se tienen en cuenta los distintos factores que median el proceso en mención, principalmente la valoración que hace el maestro de la tarea del estudiante se comprende que el logro académico, como dato numérico, no es garantía de comprensión de los contenidos de aprendizaje o de la excelencia académica del estudiante.

De igual forma, no es acertado pensar en la nota como un criterio objetivo vinculado a la realización de la tarea, por parte del estudiante, o como garante de la adquisición de condiciones personales de excelencia académica, aun cuando sea el principal criterio de reconocimiento, aprobación y tránsito por el sistema de formación, traducido como logro académico, como señala Cascón (2000, pp. 1-11).
En este sentido, la nota y por ende el logro académico se verán afectados por múltiples factores distantes de la didáctica, el estilo del maestro o el estilo de su enseñanza, y se observan con mayor relevancia los criterios de relación, significación, condescendencia o si se quiere "humanidad" 4 , como afirma uno de los docentes consultados, a la hora de valorar el trabajo realizado por el estudiante.

Por lo anterior, el contenido disciplinar tendrá un lugar relativo en el proceso de formación, obligando al docente a establecer criterios, subcriterios de valoración de su asignatura y, en ocasiones, a desplazar o sobreponer condiciones provenientes de otras dimensiones, para dar cuenta de lo que acontece con las notas del estudiante.

Una importante excepción se presenta en los espacios académicos relacionados con el área administrativa, de leguaje o ciencias biomédicas, las cuales por tradición didáctica y disciplinar tienen implícita la idea de que la valoración baja es lo normal y lo deseable para reflejar rigurosidad en el proceso de formación, presentando criterios de valoración más estrictos, que en términos estadísticos se traduce como un bajo logro académico del estudiante.

Por consiguiente, el logro del estudiante no es propiamente el resultado de lo que este hace como tarea de aprendizaje, sino la interacción entre los múltiples factores asociados a los criterios de valoración del docente, en la enseñanza.

Para concluir, desde el análisis cualitativo y cuantitativo de la información, se puede corroborar que existe una relación directa y determinante entre los criterios sociales, psicosociales y humanísticos de los estilos de enseñanza del docente y el logro académico del estudiante.

Se pudo establecer que los docentes cuya tendencia se encuentra en el estilo de enseñanza orientado hacia el aprendizaje promueven de mejor manera el logro académico del estudiante, en cuanto valoran

4 Para un importante número de los docentes consultados, la valoración de las acciones académicas del estudiante se encuentra mediada por la idea de consecuencias inmediatas en la vida del estudiante, ya sean sociales o académicoadministrativas como, por ejemplo, la posibilidad de deserción, es decir, aquello que ha de acontecer con el estudiante si el resultado del proceso de formación no es satisfactorio. 
con puntuaciones más altas las acciones, trabajos o procesos desarrollados por los estudiantes.

Se puede afirmar que el estilo de enseñanza asumido por el docente promueve el logro, si el saber fundante de este maestro es de características disciplinares (debido a sus altas valoraciones), en el caso de la licenciatura, los espacios académicos vinculados a la didáctica del deporte o de fundamentación sociohumanística.

En contraposición, se puede afirmar que el estilo de enseñanza asumido por el docente limita el logro académico, si el saber fundante de este maestro no es de características disciplinares, debido a las bajas puntuaciones frente al trabajo del estudiante, lo cual es evidente en las disciplinas de fundamento administrativo, del área de lenguajes y ciencias biológicas, como ya se había mencionado.

\section{REFERENCIAS}

Abello, D., y Hernández, C. (2013). Los estilos de enseñanza de los docentes de la Licenciatura en Educación con énfasis en Educación Especial de la Universidad Pedagógica Nacional. Revista Colombiana de Educación, 64, 309-325.

Abello, D., Hernández, C., y Hederich, C. (2011). Estilos de enseñanza en docentes universitarios, propuesta y validación de un modelo teórico e instrumental. Pedagogía y Saberes, 34, 141-154.

Bain, K. (2007). Lo que hacen los mejores profesores universitarios. Valencia, España: Universitat De Valencia, Servei De Publicacions.

Benítez, M., Giménez, M., y Osicka, R (2000). Las asignaturas pendientes y el rendimiento académico: ¿existe alguna relación? Recuperado de http://www.unne.edu. ar/unnevieja/Web/cyt/cyt/humanidades/h-009.pdf.

Calderón, A., Puentes, O., Durán, V., Alzate, R., y Barinas, G. (2012). Concepciones pedagógicas y didácticas de las políticas, planes, programas y proyectos de actividad física a nivel local y nacional. Revista Lúdica Pedagógica, 2(17), 102-113.

Calderón, A., y Alzate, R. (2010). Conocimiento profesional de los profesores de Educación Física colombianos y mexicanos, sobre actividad física. Revista Lúdica Pedagógica, 2(15), 103-115.

Carpenter, B., y Tait, G. (2001). The rhetoric and reality of good teaching: a case study across three faculties at the
Queensland University of Technology. Higher Education, 42(2), 191-203. doi:10.1023/A:1017514502456.

Cascón, L. (2000), análisis de las calificaciones escolares como criterio de rendimiento académico. Recuperado de https://campus.usal.es/ inico/investigacion/jornadas/jornada2/comun/c17.html

Castro, J., y Durán, V. (2013). Lo cierto y lo incierto del juego y la lúdica en la escuela. Revista Lúdica Pedagógica, 2(18), 21-27.

Cohen, J. H., y Amidon, E. J. (2004). Reward and punishment histories: A way of predicting teaching style? Journal of Educational Research, 97(5), 269-270. doi:10.3200/ JOER. - 97.5.269-280.

Durán, V. (2010). Cuerpo y educación en la cultura wayuu. Revista Educación Física y Deporte 29(2), 239-252.

Durán, V. (2011a). Educación física y desarrollo humano. Un nuevo horizonte para la educación del cuerpo. Revista Internacional Magisterio Educación y Pedagogía, (50), 3843.

Durán, V. (2011b) Comunidad wayuu. Educación y cultura. Revista Lúdica Pedagógica, 2(15), 169-177.

Durán, V. (2012). Antecedentes y perspectivas investigativas del conocimiento profesional docente en actividad física. Revista Lúdica Pedagógica, 2(17), 153-164.

Durán, V.H., Pedraza, A. y Serna, E. (2014). Estilos de aprendizaje y logro académico de estudiantes de licenciatura en deporte. Revista Horizontes Pedagógicos, 16, 42-54.

Durán, V., Serna, E., y Pedraza, A. (2016). Estilo de aprendizaje y logro académico en estudiantes de la Licenciatura en Deporte, Universidad Pedagógica Nacional. Informe de investigación remitido al Centro de Investigaciones Universidad Pedagógica Nacional (CIUP).

Feixas, M. (2002). El desarrollo profesional del profesor universitario como docente (Tesis doctoral). Facultad de Ciencias de la Educación, Universidad Autónoma de Barcelona, España.

Feixas, M. (2006). Cuestionario para el análisis de la orientación docente del profesor univer-sitario. Revista de Investigación Educativa, 24(1), 97-118.

Feixas, M. (2010). Enfoques y concepciones docentes en la universidad. Relieve, 16(2), 1-27. Recuperado de http://www.uv.es/RELIEVE/v16n2/RELIEVEv16n2_2. htm.

Gayle, G. (1994). A new paradigm for heuristic research in teaching styles. Religious Education, 89(1), 9-41. doi:10.1080/0034408940890102. 
Grasha, A. (1994). A matter of style: the teacher as expert, formal authority, personal model, facilitator. College Teaching, 42(4), 142-149.

Grasha, A. (Julio-agosto, 2003). The Dynamics of One-onOne Teaching. The Social Studies, 94(4), 179-187.

Heimlich, J. E., y Norland, E. (2002). Teaching style: Where are we now? New Directions for Adult \& Continuing Education, 93, 17-25. doi:10.1002/ace.46.

Hernández, V. C., y Abello, C. D. (2013). Los estilos de enseñanza de los docentes de la Licenciatura en Educación con énfasis en Educación Especial. Revista Colombiana de Educación, (64), 309-325.

Jiménez, M. (2000). Competencia social: intervención preventiva en la escuela. Infancia y Sociedad, 24, 21-48.

Laudadío, J. (2012). Evaluación de estilos de enseñanza en la universidad: estudio preliminar de las propiedades psicométricas del Cuestionario sobre la Orientación Docente del Profesor Universitario (CODPU). Interdisciplinaria, 29(1), 79-93.

Laudadío, J. (2012a). Estilos de enseñanza en la universidad. Mendoza, Argentina: Universidad Nacional de Cuyo.

Laudadío, J. (2012b). Estudio preliminar de las propiedades psicométricas del cuestionario sobre la orientación docente del profesor universitario. Interdisciplinaria, 29(1).

Laudadío, M., y Da Dalt, E. (septiembre-diciembre, 2014). Estudio de los estilos de enseñanza y estilos de aprendizaje en la universidad. Educación y Educadores, 17(3), 483-498.
Laudadío, M. y Mazzitelli, (2015). Estilos de enseñanza de los docentes de distintas carreras de nivel superior vinculadas con las ciencias naturales. Revista Educación, XXIV(46), 9-25.

Mosston, M., y Ashworth, S. (1993). La enseñanza de la educación física: la reforma de los estilos de enseñanza. Barcelona: Editorial Hispano Europea, S. A.

Pedraza, A., Durán, V., y Serna, E. (2015). Estilos de aprendizaje y logro académico de estudiantes de Licenciatura en Deporte. Revista Horizontes Pedagógicos, 16, 42-54.

Prosser, M., y Trigwell, K. (1996). Confirmatory factor analysis of the approaches to teaching inventory. British Journal of Educational Psychology, 76, 405-419.

Prosser, M., y Trigwell, K. (1999). Understanding learning and teaching: The experience in higher education. Buckingham: Society for Research Into Higher Education and Open University Press.

Saza, L., y Henao, G., (2012). Actitudes-estilos de enseñanza: su relación con el rendimiento académico. International Journal of Psychological Research, 5(1), 133-141.

Schaefer, K. M., y Zygmont, D. (2003). Analyzing the teaching style of nursing faculty. Nursing Education Perspectives, 24(5), 238.

Vaughn, L., y Baker, R. (2001). Teaching in the medical setting: Balancing teaching styles, learning styles and teaching methods. Medical Teacher, 23(1), 39-43.

Zhang, L. (2001). Approaches and thinking styles in teaching. The Journal of Psycology, 135(5), 547-561. 